\title{
EFFECT OF LIPID RATIO OF STEARIC ACID AND OLEIC ACID ON CHARACTERISTICS OF NANOSTRUCTURE LIPID CARRIER (NLC) SYSTEM OF DIETHYLAMMONIUM DICLOFENAC
}

\author{
Esti Hendradi*, Noorma Rosita, Erwinda Rahmadhanniar
}

\begin{abstract}
Department of
Pharmaceutics, Faculty of Pharmacy Airlangga University.

Jl. Dharmawangsa No.4-6, Airlangga, Gubeng, Kota SBY, Jawa Timur 60286
\end{abstract}

Submitted: $11-09-2017$

Revised: 21-10-2017

Accepted: 10-12-2017

Corresponding author Esti Hendradi

Email:

estihendradi@yahoo.com

\begin{abstract}
The aim of this study was to determine the effect of lipid ratio of stearic acid and oleic acid on the physical characteristics as well as the entrapment efficiency of diethylammonium diclofenac with Nanostructure Lipid Carrier (NLC) system. Diethylammonium diclofenac (DETA) is Non-Steroid AntiInflamatory Drugs (NSAIDs) that has been widely used in the treatment of osteoarthritis and rheumatoid arthritis. In the formulation of NLC-DETA, three different lipid ratios were used, which the ratio of stearic acid:oleic acid were 60:40, 70:30, and $80: 20$, respectively. In this NLC system, DETA served as the active drug, stearic acid as solid lipid, oleic acid as liquid lipid, and Tween 80 as surfactant components. NLC were characterized for organoleptic characteristics, $\mathrm{pH}$, viscosity, particle morphology, particle size and polydispersity index (PI), profiles of Fourier Transform Infra-Red (FTIR) and Differential Thermal Analysis (DTA), and drug entrapment efficiency. The particle shape and morphology were determined by Transmission Electron Microscopy (TEM). The results showed that the different ratios of oleic acid lipids and stearic acid had no significant effects on the viscosity and entrapment efficiency of NLC-DETA. On the other hand, it affected the $\mathrm{pH}$ of all formulas, which were significantly different. Increasing the amount of liquid lipid in the formulations reduced the size of NLC-DETA particles.
\end{abstract}

Keyword: DETA, NLC, physicochemical characterization, entrapment efficiency

\section{INTRODUCTION}

Non-Steroid Anti-Inflamatory Drugs (NSAIDs) can be classified based on the drug's selectivity to inhibit cyclooxygenase (COX) enzymes. This inhibition can reduce the synthesis of prostaglandins that leads to decrease of gastric mucosa production and triggers irritation (Gunawan et al., 2007). Diethylammonium diclofenac (DETA) is a non-selective COX inhibitor NSAID. In the oral route, the absorption of diclofenac in the intestines is fast and complete, but the bioavailability is only about $50 \%$ due to firstpass metabolism effects. Approximately $99 \%$ of diclofenac is bound to plasma phenomena (Sweetman, 2009). According to these, it is important to prepare DETA into topical dosage forms. The topical route can improve the drug efficacy and patient compliance because it is more comfortable to use and may not available for first pass metabolism (Jorge et al., 2011). DETA is a lipophilic drug with $\log \mathrm{P}$ value of 0.853 , so it can be used for topical route. The ion pair formation between anions and cations of alkilamonium diclofenac may increase skin permeability and drug solubility in non-aqueous carrier (Fini et al., 2012). An analgesic such as DETA is intended to give rapid and long term effect. Therefore, it requires a delivery system that is capable of carrying DETA in order to achieve the desired effect. Nanostructure Lipid Carrier (NLC) may become an appropriate delivery system for DETA. NLC is a nano-sized particles which the drugs are dispersed in a binary mixtures of solid lipid and liquid lipid. The nanostructures of NLC can increase the drug loading capacity (Meghana et al., 2012). NLC provides many 
advantages i.e. having low toxicity and increasing drug contact with the stratum corneum thus improving drug penetration because of its small particle size (Okyar et al., 2012). NLC consists of a mixture of solid lipid and liquid lipid that can accommodate the space needed for the entrapping drugs (Meghana et al., 2012). NLC components of the system including the solid lipid and liquid lipid. The efficacy of this carrier system is highly determined by drug entrapment efficiency, which is affected by the ratio of the solid lipid and liquid lipid and particle size (Meghana et al., 2012; Shinde et al., 2013). It has been reported that the NLC system of Q10 co-enzyme, which composed of cetyl palmitate-alpha tocopherol acetate at ratio of 70:30 (W/W), was produced in good characteristics of Q10 co-enzymeloaded NLC with high drug entrapment, good penetration, controlled-drug release, and stable, even for 90 days storage (Putranti et al., 2017). Other study reported that the meloxicamloaded NLC (MLX) was used for topical application using monostearin and alpha-tocopherol as lipid matrix. These lipids was combined at six different ratios of solid and liquid lipid matrix, which were 70:30, 75:25, 80:20, 85:15, 90:10, and 95:5, respectively. Increasing the concentration of liquid lipid in this NLC system enhanced the entrapment efficiency of MLX (Anggraeni et al., 2017).

This study aimed to investigate the characterization and entrapment efficiency of NLC loading DETA (DETA-NLC) prepared at different ratio of stearic acid and oleic acid. In addition, we further evaluated which lipid ratio that was able to produce good characterization and entrapment efficiency of DETA-NLC.

\section{MATERIALS AND METHODS}

DETA was obtained from PT. Combiphar. Stearic acid was bought from PT. Sumi Asih Oleochemicals Industry. The oleic acid was purchased from Marks \& Nos Inc. Tween 80 was found from PT. Croda. All of these chemicals were in pharmaceutical grade. The $\mathrm{Na}_{2} \mathrm{HPO}_{4} \cdot 2 \mathrm{H}_{2} \mathrm{O}$ and $\mathrm{NaH}_{2} \mathrm{PO}_{4} \cdot \mathrm{H}_{2} \mathrm{O}$ were in analytical grade and bought from Merck.

\section{Preparation of diethylammonium diclofenac loaded NLC}

Firstly, the oleic acid, stearic acid, and DETA were put in a beaker glass and melt using a hot plate (Dragon Lab MS-H Pro) at $75^{\circ} \mathrm{C}$. On the other side, a beaker glass containing aqueous phase i.e. phosphate buffer and Tween 80 was stirred by heating at $80^{\circ} \mathrm{C}$. This hot aqueous phase was then added into lipid phase and this mixture was then homogenized by using a high speed homogenizer (Ultra Turax High Shear Homogenizer) at speed of $3800 \mathrm{rpm}$ for $30 \mathrm{~min}$ in 3 cycles. The DETA-NLC was obtained by cooling the mixture at room temperature while stirring at $1500 \mathrm{rpm}$ for $30 \mathrm{~min}$.

\section{Characterization of deta-NLC}

Before characterization of DETA-NLC, the homogeneity test of each formula was evaluated by determining the recovery percentage, which was then calculated for the percentage of variation coefficient $(\% \mathrm{KV})$. It has been stated that homogenous preparation or formula was produced if the $\% \mathrm{KV}$ value is less than $6 \%$.

\section{pH}

The $\mathrm{pH}$ of DETA-NLC was measured with a digital $\mathrm{pH}$ meter (Schott Glass Mainz tipe CG 824), which was standardized using pH 6.0 standard buffers before use.The electrode was inserted into about $10 \mathrm{~mL}$ of DETA-NLC sample; the electrode must be sinked into sample. The $\mathrm{pH}$ values observed until the screen showed a stable results.

\section{Viscosity}

The viscosity of samples were measured using Brookfield Cone and Plate viscometer HDV $-Y+C P$. The cone was set at speed of $20 \mathrm{rpm}$ and about $2.0 \mathrm{~mL}$ of DETA-NLC sample was placed in the sample cup. The viscometer was then turned on and put down until the stable measurement result was achieved.

\section{Transmission electrone microscopy}

The particle morphology was determined by using Transmission Electron Microscopy (TEM JEOL JEM 1400). A drop of sample was placed on the grid, then it was stained with $2 \%(\mathrm{w} / \mathrm{v})$ of phosphotungstic acid solution. 
Table I. Composition of the DETA-NLC formulations ( $\% \mathrm{w} / \mathrm{w})$

\begin{tabular}{lccc}
\hline \multicolumn{1}{c}{ Composition } & Formula 1 (F1) & Formula 2 (F2) & Formula 3 (F3) \\
\hline DETA & 1.16 & 1.16 & 1.16 \\
Stearic acid & 6 & 7 & 8 \\
Oleic acid & 4 & 3 & 2 \\
Tween 80 & 5 & 5 & 5 \\
\hline
\end{tabular}

After staining process, the samples were dried at room temperature. Once the samples were ready, then they were observed with TEM.

\section{Measurement of particle size and Polydispersity Index (PDI)}

The analysis was performed at $25^{\circ} \mathrm{C}$ with detection angle of $165^{\circ} \mathrm{C}$ using a dynamic light scattering instrument (Delsa ${ }^{\mathrm{TM}}$ Nano Submicron Particle Size and Zeta Potential Dynamic Light Scattering). The particle size was calculated from the fluctuation of the average intensity of light scattering. The PDI shows the homogenity of particle size within nanoparticles population.

\section{Differential thermal analysis}

The thermal Analysis of stearic acid, DETA, and DETA-NLC were perfomed using Differential Thermal Analyzer (DTA SP 900 Thermal System Metler Toledo SP 85). All the samples were heated in aluminum pans. The analysis was performed with a heating range of $30-200^{\circ} \mathrm{C}$ and at a hating rate of $5^{\circ} \mathrm{C} / \mathrm{min}$.

\section{FTIR Spectroscopy}

The FT-IR analysis helps to confirm the identity of the drug and to detect the interaction between the drug and carriers. The FT-IR spectral instrument (IR JASCO FT/IR5300 Instrument) was used for measurement of native DETA, single component of NLC, and DETA-NLC. The samples were analyzed at ambient temperature. All the spectra acquired were scanned between 400 and $4000 \mathrm{~cm}^{-1}$

\section{The drug quantification assay and entrapment efficiency}

About $0.1 \mathrm{~g}$ of DETA-NLC was dissolved in a $10 \mathrm{~mL}$ of phosphate buffer $\mathrm{pH}$ 6.0 \pm 0.1 . This mixture was then centrifuged (Hettich Rotofix 32) at 2500rpm for $30 \mathrm{~min}$, and then filtered through $0.45 \mu \mathrm{m}$ filter paper to obtain free drug that contained in the supernatant. The concentration of DETA in the supernatant was then determined by UV-VIS spectrophotometer (Double beam Spectrophotometer UV-1800 Shimadzu) using three wavelength methods, which were $266 \mathrm{~nm}$, $276 \mathrm{~nm}$, and $286 \mathrm{~nm}$ with appropriate dilutions. The Entrapment efficiency was calculated using following equation.

$\% \mathrm{EE}=\frac{W a-W s}{W a} \times 100 \%$

which EE refers to entrapment efficiency, Wa stands for the weight amount of DETA added to the formulation, and Ws is the quantified weight of DETA in the supernatant.

\section{Statistical analysis}

The characterization of DETA-NLC system, which includes $\mathrm{pH}$, viscosity, particle size, and entrapment efficiency of drug, was statistically analyzed using a one-way analysis of variance (ANOVA) method at a 95\% confidence interval. Then, the results were further analyzed using honestly significant difference (HSD) test.

\section{RESULT AND DISCUSSION Preparation of NLC diethylammonium diclofenac}

It has been known that the melt emulsification followed by high speed homogenization is a reliable, simple and reproducible method for preparation of NLC. In this study, the high speed homogenization of the lipid phase with hot aqueous phase containing surfactant such as Tween 80 at speed of $3800 \mathrm{rpm}$ for $10 \mathrm{~min}$ was sufficient to produce an emulsion with average particle size between 351.9-1370.0nm. The homogeneity test results showed that the DETA recovery 
Table II. The pH and viscosity of DETA-NLC

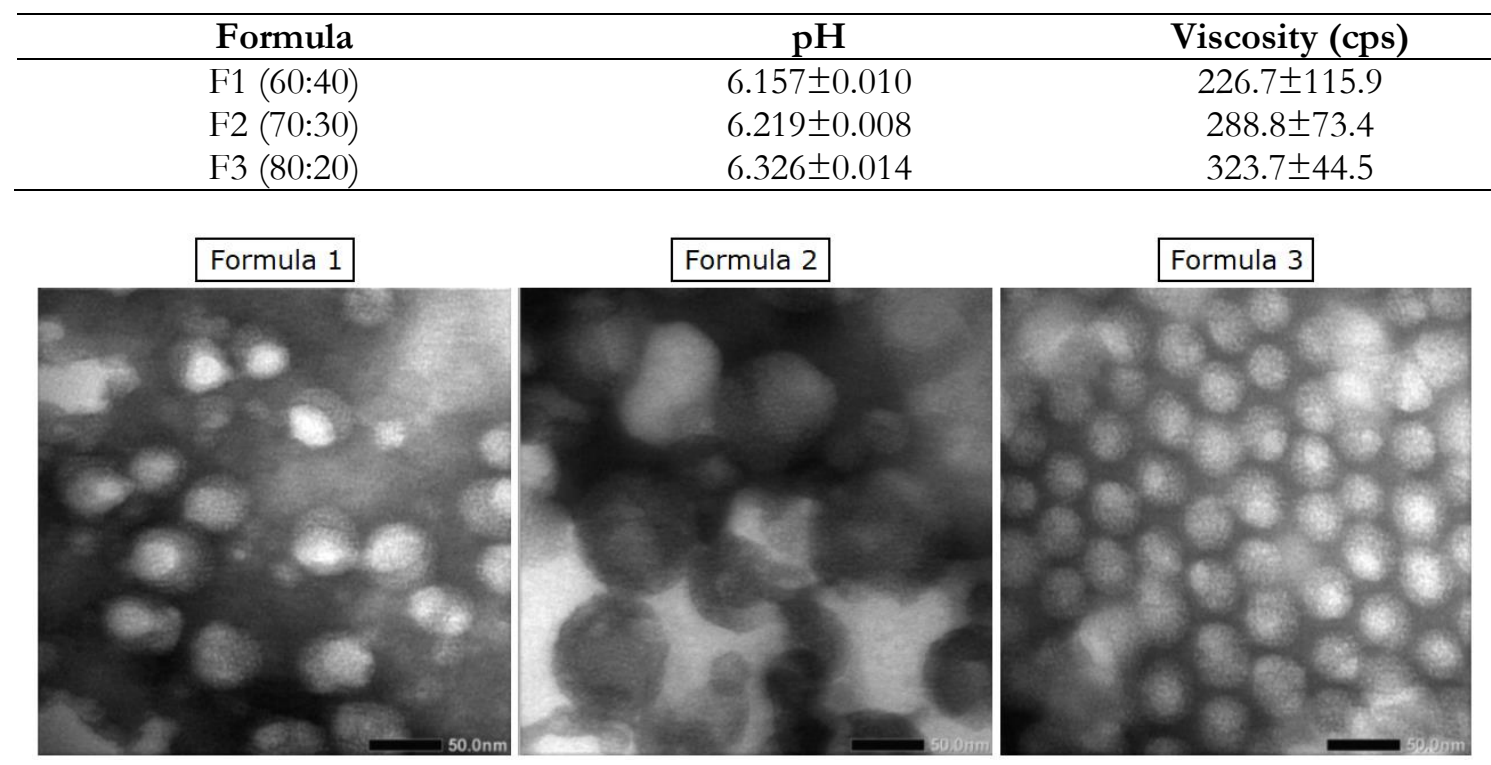

Figure 1. Transmission electron photomicrograph of F1(60:40, Formula 1), F2 (70:30, Formula 2), and F3 (80:20, Formula 3) of DETA-NLC at magnification of $80000 \mathrm{X}$.

percentage of F1, F2, and F3 of DETA-NLC were $107.2 \%, \quad 106.9 \%$, and $106.3 \%$, respectively. Moreover, the percentage of $\mathrm{KV}$ of F1, F2 and F3 were $3.35 \%, 0.97 \%$, and $1.46 \%$, respectively (Table I).

pH

The $\mathrm{pH}$ of the all formula was found about 6.157 to 6.326 (Table II). It could be suggested that DETA-NLC can be used for topical application without producing skin irritation. Based on the statistical analysis of ANOVA followed by HSD test, the results showed that there were significant difference of $\mathrm{pH}$ values among all formula, which were $\mathrm{F} 1<\mathrm{F} 2<\mathrm{F} 3$. These differences may be due to the different amount of oleic acid used in NLC. Increasing oleic acid reduced the $\mathrm{pH}$ value of the DETA-NLC.

\section{Viscosity}

The viscosity of the sample is intended to enable NLC adhering onto the skin surface, thus increasing the residence time and drug penetration across the skin. Thereby, it can improve drug availability at site of action by a controlled-manner and avoid systemic absorption. As (Table II), the results showed no significance differences of viscosities among all formulas $(\mathrm{P}>0.05)$. It indicates that different ratio of stearic acid and oleic acid produced no effects on the viscosity of the DETA-NLC.

\section{Transmission electrone microscopy}

The particle morphology of NLC was evaluated with JEOL JEM 1400 TEM electron microscope at a magnification of 80.000times. The photomicrograph showed that there were spherical forms observed that corresponds to NLC particles (Figure 1). The observed particle size was less than $100 \mathrm{~nm}$. However, the NLC particles of F1 and F2were seen separately and not clustered. While on the F3, they attached each other.

\section{Particle size and PDI}

The optimized NLC formula showed small particle size and the value of polydispersity index (PDI) of all formula were found to be $<1.0 \mu \mathrm{m}$. It suggested that the optimized NLC formula have particle size in nanometer range and monodispered system, which remains stable and will not convert macrometer range particles. As the result, F1 showed the smallest particle size. On the other hand, F3 had a micrometer size that may 
because of the low amount of oleic acid. The greater amount of liquid lipid, the smaller the particle size of the NLC could be achieved. At a ratio of stearic acid and oleic acid of 80:20, respectively, it would require large amounts surfactants to stabilize the system and prevent aggregation of lipid particles. In this study, the particle size that was measured by TEM was smaller than that of measured by DLS. It may be due to the effect of the dilution process during particle size measurement using DLS. PDI reflects the particle homogeneity and it varies from 0.0 to 1.0. The closer the value of PDI to 0 , the more homogenous the particles. Increasing the solid lipid has been reported to cause the particle aggregation (Phatak and Chaudhari, 2013). F1 and F2 met the characteristics of the NLC system, which had sizes in nanometer range $(<1 \mu \mathrm{m})$ (Table III). While the F3 did not met the specification because of its micrometer size $(>1 \mu \mathrm{m})$. It might be due to the high amount of stearic acid and low amount of oleic acid of F3. At the ratio of stearic acid and oleic acid of 80:20, respectively, it requires a high amount of surfactant to stabilize the system and prevent the incorporation or aggregation of lipid particles. The surfactant was used to stabilize the nanoparticles and prevent the incorporation of particles (Pezeshki et al., 2014). To decrease the particle size of F3, a cosurfactant could be added as the system stabilizer, for preventing aggregation of lipid particles

Table III. The particle size and Poly Dispersity Index(PDI) of DETA-NLC

\begin{tabular}{lcc}
\hline Formula & Particle Size (nm) & PDI \\
\hline F1 (60:40) & $351.9 \pm 34.8$ & $0.373 \pm 0.039$ \\
F2 (70:30) & $518.1 \pm 92.3$ & $0.412 \pm 0.030$ \\
F3 (80:20) & $1370.0 \pm 326.5$ & $0.489 \pm 0.032$ \\
\hline
\end{tabular}

Based on one-way ANOVA analysis, there was significant difference of particle size of DETA-NLC $(\mathrm{P}=0.002)$. It was further analyzed using HSD test and the results showed that F1 (60:40) had particle sizes diameter that were similar to F2 (70:30). On the other hand, F3 (80:20) had significantly different particle size compared to F1 and F2. Moreover, the results of TEM analysis supported these results. F3 showed the largest particle size and PDI among the three formulas. It could be suggested that the lipid particles of F3 have a tendency to aggregate. Therefore, the particles would easily attach each other forming large sized-particles. It was confirmed by the high PDI value of F3, which represented the non-homogeneous size distribution of F3 NLC particles.

\section{Differential Thermal Analysis (DTA)}

DTA was performed to determine the melting point of NLC-DETA and its components. In this study, native DETA has a melting point of $155.3^{\circ} \mathrm{C}$ (Figure 2). After it was prepared into NLC-DETA, DETA peak did not appear on the entire formula. The shifting peak of DETA showed that it has been trapped inside the NLC system. There was a large peak seen in the all the formula, which was within $110-114^{\circ} \mathrm{C}$. Due to the interaction among the NLC components, it might produce the polymorphism of stearic acid. It could be triggered by the addition of about 1-5\% sorbitan esters surfactants, such as Span and Tween.

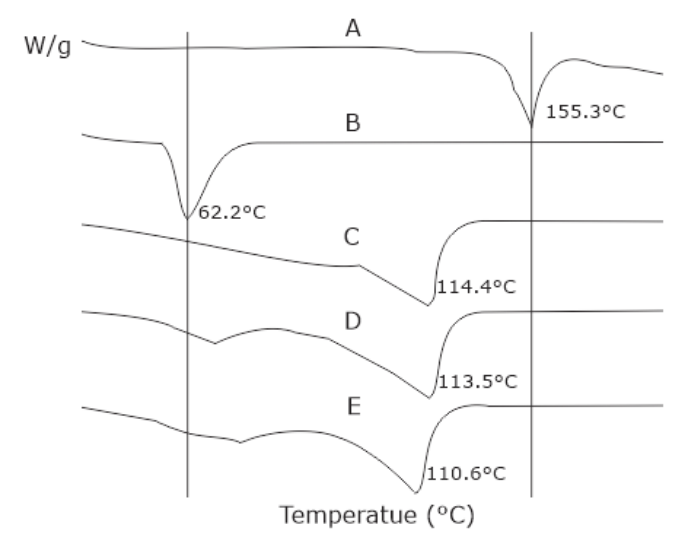

Figure 2. The profiles of differential thermal analysis of DETA (A), stearic acid (B), F1 (60:40, C), F2 (70:30, D), and F3 (80:20, E) of DETA-NLC.

\section{FT-IR Spectroscopy}

The FT-IR spectroscopy was carried out to evaluate the compatibility between native DETA with stearic acid, oleic acid, and Tween 80 that have been used to prepare the NLC. The native DETA had characteristic IR peaks at $3588.27 \mathrm{~cm}^{-1}(\mathrm{~N}-\mathrm{H}) ; \quad 744.26 \mathrm{~cm}^{-1} \quad(\mathrm{C}-\mathrm{Cl})$; 
$1689.47 \mathrm{~cm}^{-1} \quad(\mathrm{C}=\mathrm{C}) ; 2992.25 \mathrm{~cm}^{-1} \quad\left(\mathrm{C}-\mathrm{H} \quad s p^{3}\right)$. (Figure 3E, F, and G) There were no differences in the absorption. It indicates that the different ratio of stearic acid and oleic acid used in these three NLC formulas did not produce any changes on their chemical structures and there was no chemical interaction between DETA and NLC components.

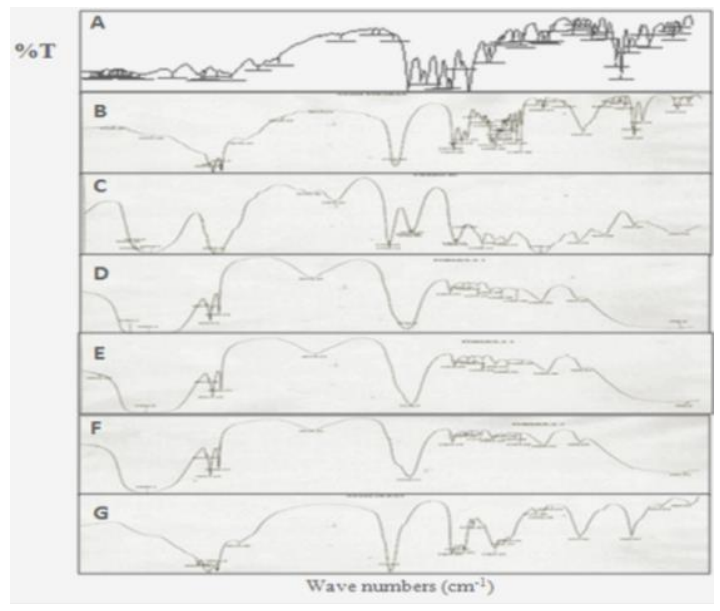

Figure 3 The profiles of IR spectra of DETA (A), Stearic Acid (B), Oleic Acid (C), Tween 80 (D), F1 (E), F2 (F), and F3 (G) of DETANLC in wave numbers $400-4000 \mathrm{~cm}^{-1}$.

\section{Entrapment efficiency}

The results indicate that the lipid concentration had critical effects on the entrapment efficacy of DETA. It has been known that the drug entrapment efficiency is lower for the sample with lower lipid concentration. The values of drug entrapment efficiency (Table IV). A higher concentration of liquid lipid resulted in larger particle size that would affect the amount of DETA entrapped inside of NLC. It is because the higher amount of liquid lipid was available to dissolve more drug molecules at formula with high lipid concentration. In addition the DETA entrapment is also affected by the solubility of DETA in the lipid component. DETA has good solubility in oleic acid, which was about $413.33 \pm 21.27 \mathrm{mg} / \mathrm{mL}$ oleic acid. The high solubility of drugs in liquid lipids and solid lipids can improve the efficiency of the entrapment. (Hussain Shah et al, 2012; Patel et al., 2012). The statistical analysis showed that there was no significant difference between the formulas of NLC system.
Table IV. The entrapment efficiency of DETA of DETA-NLC

\begin{tabular}{cc}
\hline Formula & EE (\%) \\
\hline F1 $(60: 40)$ & $73.858 \pm 0.840$ \\
F2 $(70: 30)$ & $73.654 \pm 2.079$ \\
F3 $(80: 20)$ & $73.172 \pm 1.488$ \\
\hline
\end{tabular}

\section{CONCLUSION}

In this study, it can be concluded that the different ratios of oleic acid and stearic acid, which were 60:40, 70:30, 80:20, respectively, did not affect the sample viscosity. However, there was significantly different in the $\mathrm{pH}$ value of samples for all formulas. For samples particle size and PDI, the differences were occurred for formula with lipid ratio of 80:20 to 60:40 and 70:30. The differences of entrapment efficiency were negligible for all formulas.

\section{ACKNOWLEDGEMENT}

The authors thank to Faculty of Pharmacy Airlangga University for support during this study.

\section{REFERENCES}

Anggraeni, Y., Haryanto, IY., Hendradi, E., 2017. Physical and chemical characteristics of meloxicam from nanostructured lipid carriers system using some concentration ratios of monostearin and alpha-tocopherol acetate lipid matrix. Asian J Pharm Clin Res, Vol 10, Issue 2, 2017, 132-137

Fini, A., Bassini, G., Monastero, A., Cavallari, C., 2012. Diclofenac Salts, VIII. Effect of the Counterions on the Permeation through Porcine Membrane from Aqueous Saturated Solutions. J Pharmaceutics, Vol. 4, p. 413-429.

Gunawan, SG., Setiabudy, R., Nafrialdi, \& Elysabeth., 2007. Farmakologi dan Terapi, Edisi 5, Jakarta: Balai Penerbit FKUI, hal 230-233.

Hussain Shah, SN., Salman, M., Ahmad, M., 2012. Effect of Oleic Acid on the Permeation Kinetics of Diclofenac Diethylamine. I Chem Soc Pak, No.1, Vol. 34

Jorge, LL., Feres, CC., Vitor, EP., 2010. Topical Preparations for Pain Relief: Efficacy 
and Patient Adherence. J Pain Res, Vol. 4, p. 11-24.

Meghana, SK., Vaidya, KK., Bhosale, AV., Chaundari, PD., 2012. Solid Lipid Nanoparticles and Nano Structure Carriers : An Overview. Int J Pharm Chem Bio Scie. Vol. 2(4), p. 681-691.

Okyar, A., Özsoy, Y., Güngör, S., 2012. Novel Formulation Approaches for Dermal and Transdermal Delivery of NonSteroidal Anti-Inflamatory Drugs. Intech Open Scince Mind.

Patel, D., Dasgupta, S., Dey, S., Ramani, Y. R., 2012. Nanostructured Lipid Carriers (NLC)-Based Gel for the Topical Delivery of Aceclofenac: Preparation, Characterization, and In Vivo Evaluation. Scientia Pharmaceutica, p. 749-764.

Phatak, AA. Chaudari, PD., 2013. Development and evaluation of nanostructured Lipid
Carrier (NLC) Based Topical Delivery of an Anti-Inflammatory Drug. J Pharm Res, p. $677-685$

Putranti, AR., primaharinastiti, R., Hendradi, E., 2017. Effectivity and physicochemical stability of nanostructured lipid carrier coenzyme Q10 in different ratio of lipid cetyl palmitate and alpha tocopheryl acetate as carrier. Asian J Pharm Clinical Res, Vol 10, Issue 2, 2017, 1-7.

Shinde, G., Rajesh, KS., Prajapati, N., Murthy, RSR., 2013. Formulation, Development and Characterization of Nanostructured Lipid Carrier (NLC) Loaded Gel for Psoriasis. Scholar Research Library, p. 1325.

Sweetman, SC., 2009. Martindale The Complete Drug Reference, 36 th ed., London : Pharmaceutical Press (PhP), p. 17, 44-47, 59. 